\title{
Inflammatory response to infectious pulmonary injury
}

\author{
C. Delclaux*,\#, E. Azoulay ${ }^{\mp, \#}$
}

\begin{abstract}
Inflammatory response to infectious pulmonary injury. C. Delclaux, E. Azoulay. (C) ERS Journals Ltd 2003.

ABSTRACT: This review describing the inflammatory response to infectious pulmonary injury is focused on the innate immunity of the distal lung to bacterial pneumonia. The fact that the inflammatory response varies to some extent with the bacterial strain responsible for the infection is emphasised. The key cellular components present in the distal lung are described. The major role of alveolar macrophage is described, inasmuch as it responds to the usual daily challenges of bacteria entering the terminal airways and is capable of initiating an inflammatory reaction if the microbial challenge is either too large or too virulent. Under these conditions, the alveolar macrophages initiate an inflammatory response that recruits large numbers of neutrophils into the alveolar spaces.
\end{abstract}

The strategy of the innate immune response may not be to recognise every possible antigen, but rather to focus on a few, highly conserved structures present in large groups of microorganisms. These structures are referred to as pathogen-associated molecular patterns and the receptors of the innate immune system that evolved to recognise them are called pattern-recognition receptors.

The soluble factors in innate defence, such as cytokines, are described, and a last paragraph discusses whether a specific inflammatory response could characterise nosocomial pneumonia.

Eur Respir J 2003; 22: Suppl. 42, 10s-14s.
*Service de Physiologie-Explorations Fonctionnelles, Hôpital Henri Mondor, Assistance Publique, Hôpitaux de Paris (AP-HP), Créteil, ${ }^{\#}$ Unité INSERM U492-Université Paris XII, Faculté de Médecine, Créteil, "Service de Réanimation Médicale, Hôpital Saint-Louis, AP-HP, Paris, France.

Correspondence: C. Delclaux, Service de Physiologie-Explorations Fonctionnelles, Hôpital Henri Mondor, 51, avenue du Maréchal de Lattre de Tassigny, 94010 Créteil, France.

Fax: 33148981777

E-mail: christophe.delclaux@creteil.inserm.fr

Keywords: Alveolar macrophage

bacteria

cytokine

innate immunity

neutrophil
The lung is the largest epithelial surface area of the body in contact with the external environment. The upper and lower airways are repeatedly exposed to a multitude of airborne particles and microorganisms. Since these agents are frequently deposited on the surface of the respiratory tract, an elaborate system of defence mechanisms maintains the sterility of the distal lung. This highly integrated pulmonary defence system includes the acute inflammatory phagocytic (alveolar macrophage and neutrophil) system, the mucociliary escalator apparatus, humoral immune mechanisms, including specific antibodies and nonspecific antibacterial factors, and cellular immune mechanism.

Two types of responses to invading microbes represent the total immunological capability of the host [1, 2]. Innate (natural) responses occur to the same extent however many times the infectious agent is encountered, whereas acquired (adaptative) responses improve on repeated exposure to a given infection. The innate responses use phagocytic cells (neutrophils, monocytes, macrophages), cells that release inflammatory mediators (basophils, mast cells, and eosinophils), and natural killer cells. The molecular components of innate responses include complement, acute-phase proteins, and cytokines. Acquired responses involve the proliferation of antigen-specific B- and T-cells, which occurs when the surface receptors of these cells bind to antigen. Specialised cells, called antigen-presenting cells, display the antigen to lymphocytes and collaborate with them in the response to the antigen. B-cells secrete immunoglobulins (Ig), the antigen-specific antibodies responsible for eliminating extracellular microorganisms. T-cells help B-cells to make antibody and can also eradicate intracellular pathogens by activating macrophages.

This review will focus on the inflammatory response (innate immunity) of distal lung to bacterial pneumonia (for other reviews see $[3,4])$.

\section{Cellular components present in the distal lung}

Innate immunity: the successful response of the alveolar macrophage

It is generally recognised that the rate of removal of particles from alveolar surfaces by mechanical means is very slow, in contrast to the more rapid tracheobronchial mucociliary system. It is the success of alveolar macrophage activity that determines the primary effectiveness of the immune response, even if its activity may be enhanced by the secondary arm of immunity (e.g. opsonins or cytophilic antibody). Macrophages constitute $\sim 85 \%$ of all alveolar inflammatory cells retrieved by bronchoalveolar lavage [5]. Macrophages have receptors for antibodies and complement (opsonins), which both enhance phagocytosis. The engulfed microorganisms are subjected to a wide range of toxic intracellular molecules, including superoxide anion, hydroxyl radicals, hypochlorous acid, nitric oxide (a main component), antimicrobial cationic proteins and peptides, and lysozyme. The next step is a role in antigen-processing, and in stimulating lymphocyte functions (first step of acquired immune response).

In the case of normal flora and the common airborne bacteria (as staphylococci, micrococci) there is an adequate supply of "natural" opsonins available and these organisms are readily phagocytosed. The major functional opsonins in the normal lung are probably small quantities of $\mathrm{IgG}$, and the presence of collectins (as surfactant proteins (SP): SP-A and 
SP-D [6]). Other major opsonins are complement components; however, their level in normal lung secretions is extremely low. To overcome this relative weak availability of opsonins, their supply when bacterial infection occurs is necessary. Along this line, local inflammatory reactions in the lung can cause sufficient exudation to bring in plasma antibodies and necessary complement components [7]. By contrast with common airborne bacteria, it seems that some other bacteria are able to resist phagocytosis, unless specific opsonins are present, such as Hemophilus influenzae or Pseudomonas aeruginosa. As a consequence, inflammatory response varies to some extent with the bacterial strain responsible for the infection (maybe even with the serotype involved).

Phagocytes also remove the body's own dead or dying cells. Dying cells in necrotic tissue release substances that trigger an inflammatory response, whereas cells that are dying as a result of apoptosis (programmed cell death) express molecules on their cell surface that identify them as candidates for phagocytosis, thereby restricting inflammatory reaction. This apoptotic process is strikingly important when considering neutrophils, which contain high levels of proteinases [8].

Alveolar macrophages are avidly phagocytic and readily kill ingested organisms. In addition to their phagocytic capabilities, they play a prominent role in orchestrating inflammatory and immune responses. Their direct stimulation by bacterial products or indirect stimulation via the stress response leads to the secretion of pro-inflammatory cytokines that are under the control of the transcription factors of the nuclear factor- $\kappa \mathrm{B}$ family. These cytokines are interleukin (IL)-8, IL-1 $\beta$, and tumour necrosis factor (TNF)- $\alpha$. The activation of this transcription factor in alveolar macrophages has been demonstrated during acute respiratory distress syndrome (ARDS) [9]. Moreover, previous studies in ARDS had demonstrated the increased expression in alveolar macrophages of related cytokines, namely IL- $8, \mathrm{TNF}-\alpha$, and IL-1 $\beta$ [9]. The two latter cytokines share a lot of properties; TNF- $\alpha$ has been designated as an early response cytokine [10]. TNF- $\alpha$ exerts a beneficial role during pneumonia due to its properties. First, the upregulation of adhesion molecules contributes to recruit neutrophils, which is supported by studies demonstrating neutrophil influx inhibition using neutralisation of endotoxin-induced lung TNF- $\alpha$ by either an anti-TNF- $\alpha$ antibody or a replication-deficient adenovirus encoding a soluble TNF- $\alpha$ receptor [11]. Second, TNF- $\alpha$ increases both endothelial and epithelial permeability independently of the presence of neutrophils [12], allowing the access of plasma with proteins like opsonins into alveolar space, and finally TNF- $\alpha$ increases liquid clearance from airspaces [13], the resulting balance being the sole availability of proteins. All these beneficial effects are further demonstrated by experiments using neutralisation of the TNF- $\alpha$ response, which has been shown to impair pulmonary host defences against a wide range of other pathogens, including $P$. aeruginosa, Legionella pneumophila, K. pneumoniae, Staphylococcus aureus, Streptococcus pneumoniae, and Mycobacterium tuberculosis [4]. Thus, it is now widely recognised that TNF- $\alpha$ is a pivotal mediator in determining the outcome of a broad array of infectious diseases in the host.

While these pro-inflammatory mediators (TNF- $\alpha$ and IL$1 \beta)$ are not thought to have direct chemoattractant activity, they are potent inducers of IL- 8 production by several cell types, including alveolar macrophages, type II epithelial cells, and lung fibroblasts. Among this family of chemotactic cytokines, or chemokines, IL-8 has been identified as the major chemotactic factor for neutrophils in the lung $[9,10]$.

Concomitantly to the production of pro-inflammatory cytokines, alveolar macrophages are able to synthesise antiinflammatory cytokines such as IL-10. IL-10 is a cytokine that was first recognised for its role in promoting $\mathrm{T}$-helper cell (Th) type 2 immune responses through the inhibition of cellmediated (Th1) immune responses. It is now clear that IL-10 is also important in the innate immune response to bacterial pathogens [4]. This anti-inflammatory cytokine downregulates the production of TNF- $\alpha$, interferon (IFN)- $\gamma$, and certain chemokines. For instance, VAN DER POLL et al. [14] showed that intranasal administration of $S$. pneumoniae results in a marked increase in IL-10 in the lungs of normal mice. In this model, prior intrapulmonary administration of IL-10 to the $S$. pneumoniae challenge reduced the lung TNF- $\alpha$ and IFN- $\gamma$ responses. These animals experienced higher lung and blood bacterial counts and increased early mortality.

In summary, alveolar macrophages respond to the usual daily challenges of bacteria entering the terminal airways and are capable of initiating an inflammatory reaction if the microbial challenge is either too large or too virulent to be contained by the macrophages alone. Under these conditions, the alveolar macrophages initiate an inflammatory response that recruits large numbers of neutrophils into the alveolar spaces. Substances capable of eliciting the immigration of these neutrophils into the airways include complement components, arachidonic acid metabolites such as leukotriene $\mathrm{B}_{4}$, and chemotactic peptides such as IL-8 and related chemokines. An unresolved issue is whether alveolar and interstitial macrophages have different functions. It seems that interstitial macrophages are more likely to secrete mediators, as cytokines, than the former, which could be more involved in bactericidal activity.

\section{Neutrophils}

When the microbial challenge is either too large or too virulent, large numbers of neutrophils are recruited into the alveolar spaces from the marginated pool of neutrophils in the pulmonary vasculature [15]. As a matter of fact, neutrophils are virtually absent from alveolar space in a healthy condition. In the lung, neutrophil emigration occurs at the capillary level, by contrast with its veinule emigration in other organs. Substances released from the pathogen and from damaged tissues upregulate the expression of adhesion molecules on vascular endothelium, alerting passing cells to the presence of infection. The cell-surface molecule L-selectin on neutrophils recognises carbohydrate structures on the vascular adhesion molecules. The neutrophil rolling along the vessel wall is arrested in its course by these interactions (this latter effect could be absent in the lung due to the small size of capillaries inducing physical neutrophil arrest). As the neutrophil becomes activated, it rapidly sheds L-selectin from its surface and replaces it with other cell-surface adhesion molecules such as the integrins. These integrins bind the molecule E-selectin, which appears on the blood vessel wall under the influence of inflammatory mediators such as bacterial lipopolysaccharide and the cytokines interleukin- $1 \beta$ and TNF- $\alpha$. Interestingly, depending on the bacterial strain involved, mechanisms of neutrophil emigration can differ, at least in terms of the kind of adhesion molecule involved. Then, the activated neutrophils pass through the vessel walls, moving up the chemotactic gradient to accumulate at the site of infection. Neutrophils also have receptors for opsonins allowing enhancing phagocytosis. The engulfed microorganisms are subjected to a wide range of toxic intracellular molecules, including superoxide anion, hydroxyl radicals, hypochlorous acid, nitric oxide (less importantly), antimicrobial cationic proteins and peptides, and lysozyme. Neutrophils also participate in the inflammatory response via secretion of pro-inflammatory cytokines such as TNF- $\alpha$ and IL-1 $\beta$ (neutrophil cytokines 
are reviewed in [16]). However, neutrophils are unable to synthesise the anti-inflammatory cytokine, IL-10.

\section{Dendritic cells}

A key cellular component of innate immunity is the interdigitating dendritic cell that constantly but quietly endocytoses extracellular antigens [1]. However, they become activated and behave as antigen-presenting cells when pattern-recognition receptors on their surface recognise distinctive pathogenassociated molecular patterns on the surface of microorganisms.

Along this line, the strategy of the innate immune response may not be to recognise every possible antigen, but rather to focus on a few, highly conserved structures present in large groups of microorganisms [17]. These structures are referred to as pathogen-associated molecular patterns (for instance, bacterial lipopolysaccharide, peptidoglycan, lipoteichoic acids), and the receptors of the innate immune system that evolved to recognise them are called pattern-recognition receptors. The receptors of the innate immune system that are encoded in the germline differ from antigen receptors in several ways. They are expressed on many effector cells of the innate immune system, most importantly on macrophages, dendritic cells, and B-cells (the professional antigen-presenting cells), but also on other cells such as epithelial cells. These receptors belong to three classes: secreted, endocytic, and signalling. Secreted pattern-recognition molecules function as opsonins, the best characterised is the mannan-binding lectin. This latter receptor forms a structurally related family of collectins with surfactant proteins. Endocytic pattern-recognition receptors occur on the surface of phagocytes. Signalling receptors recognise pathogen-associated molecular patterns and activate signal-transduction pathways that induce the expression of a variety of immune-response genes, including inflammatory cytokines. The recently identified receptors of the toll family appear to have a major role in the induction of immune and inflammatory responses.

\section{Natural killer cells}

Natural killer cells destroy infected cells via antibodydependent cellular cytotoxicity [1]. Inserting the pore-forming molecule perforin into the membrane and then injecting with cytotoxic granzymes kills the targeted cell. Recently, HASHIMOTO et al. [18] have demonstrated an increased expression of messenger ribonucleic acid (mRNA) of these mediators in bronchoalveolar lavage fluid cells obtained from ARDS patients.

\section{Alveolar epithelial cells}

It has been demonstrated that alveolar epithelial cells actively participate in the inflammatory response during bacterial infection [19]. Indeed, these cells are able to synthesise acute phase proteins (such as C-reactive protein and inhibitors of proteases), chemokine (such as IL-8) and cytokines. These cells are activated by alveolar macrophage products, such as TNF- $\alpha$, which increases their permeability. This increase in permeability allows soluble CD14 to reach airways; alveolar epithelial cells, which lack membrane CD14, can then be responsive to the bacterial product, endotoxin.

In summary, all cells that are present in alveoli participate to some extent in the inflammatory response of innate immunity. The main cellular components of the innate immune response, the alveolar macrophage and the neutrophil, need to communicate with each other if an effective host defence is to be mounted. Mechanisms are needed to initiate this response, but also to localise, reinforce, and ultimately resolve it. One of the essential components of the immune system that plays a critical role in these processes are the soluble factors of innate defence.

\section{Soluble factors in innate defence}

Innate responses frequently involve complement, acutephase proteins, and cytokines [1]. Nitric oxide has been more recently shown to be one of the major actors of the immune response.

\section{Complement activation}

The early events of complement activation can be triggered by one of three pathways. The classic pathway is activated by antigen-antibody complexes, the alternative pathway by microbial-cell walls, and the lectin pathway by the interaction of microbial carbohydrates with mannose-binding protein in the plasma. The outcome is the generation of opsonins (the $\mathrm{C} 3 \mathrm{~b}$ molecule), chemoattractant (C5a) or membrane-attack complex (C5b, C6, C7, C8 and C9), which perforates cell membranes leading to cell death.

\section{Acute-phase proteins}

The molecules collectively referred to as acute-phase proteins enhance resistance to infection and promote repair of damaged tissue. In addition to some complement components, the acute phase proteins include $\mathrm{C}$-reactive protein, serum amyloid A protein, proteinase inhibitors, and coagulation proteins.

\section{Cytokines}

Cytokines constitute another group of soluble mediators [10]. Cytokines that have been shown to fulfil an important role in the innate immunity of the lung include TNF- $\alpha$, IL-10, IL-12, IL-6, the chemokines (IL-8), IFN- $\gamma$ and granulocyte colony-stimulating factor (G-CSF). The role of some of them has been previously detailed (see above).

Numerous studies have shown that IL-12 can enhance cellmediated host resistance to a wide range of intracellular pathogens. Utilising immunohistochemical techniques in animals infected with $K$. pneumoniae, researchers have found that IL-12 production appears to be localised primarily to alveolar macrophages, pulmonary epithelial cells, and neutrophils [20].

Monocytes, macrophages, lymphocytes, fibroblasts and even alveolar epithelial cells produce IL-6 [10]. It stimulates the B-cell Ig production, T-cell proliferation, natural killer cell activation and cytotoxicity. It also plays a role in the increase in body temperature and hepatic production of acute phase proteins such as $\mathrm{C}$-reactive protein and inhibitors of proteinases.

IFN- $\gamma$ is a glycoprotein derived from activated T-lymphocytes with effects on macrophages, monocytes, neutrophils, endothelial and epithelial cells and fibroblasts. Overall, IFN- $\gamma$ activates those cells that act as a defence mechanism against invading bacteria $[3,4,11]$.

For more than a century, physicians have recognised the relationship of the white blood cell count to the occurrence, severity, and outcome of many infectious diseases. Colonystimulating factors are a family of acidic glycoproteins that are required for the proliferation and differentiation of haematopoietic progenitor cells. Of this cytokine family, which 
includes granulocyte macrophage colony-stimulating factor, macrophage colony-stimulating factor, IL-3, and G-CSF, it is G-CSF that plays an important role in maintaining the normal blood neutrophil count and enhancing the functional properties of neutrophils, including chemotaxis, phagocytosis, and bactericidal activity. Mononuclear phagocytes, including alveolar macrophages, are known to produce G-CSF when stimulated by bacterial products or cytokines. TAZI et al. [21] reported that alveolar macrophages recovered from patients with pneumonia produce G-CSF spontaneously, whereas alveolar macrophages from healthy control subjects produce G-CSF only after endotoxin stimulation. G-CSF acts locally to activate and recruit neutrophils into the infected lung and functions systemically to stimulate the formation of additional neutrophils, thus reinforcing the host's response until the infection is resolved $[3,4,11]$.

\section{Nitric oxide: an additional mediator of innate inflammatory response}

The role of nitric oxide in the immune response has been the subject of a recent review [22]. Nitric oxide is one of the major mediators of innate immune response due to its involvement in alveolar macrophage bactericidal activity and in neutrophil migration ability. Moreover, nitric oxide could participate in the regulation of cytokine synthesis by alveolar macrophages. Nitric oxide has broad-spectrum antimicrobial properties, and is synthesised by the epithelium of the respiratory tract together with inflammatory cells. Consequently, nitric oxide diffuses in airway gas and can therefore be measured in this exhaled gas [23]. The high concentration of nitric oxide in proximal airways, as sinus cavities, is supposed to participate in the maintenance of their sterility. During infectious processes of the respiratory tract an increase in exhaled nitric oxide is evidenced, probably due to both increased output from both epithelium and inflammatory cells (since their inducible nitric oxide synthase is upregulated by endotoxin).

\section{Localised versus generalised inflammatory response}

Using bronchoalveolar lavage evaluation in human beings, almost all soluble mediators of innate immune response have been assessed during bacterial pneumonia. In this latter setting, a localised inflammatory response is usually evidenced (compartmentalisation in the lung area involved [24]) at least in terms of cytokine secretion. An exception is the usual increase in IL-6 in both bronchoalveolar lavage fluid and plasma (to induce hepatic synthesis of acute phase proteins) [3].

The accurate balance of pro/anti-inflammatory response of alveolar macrophages is probably one of the main determinants of compartmentalisation of lung infection. This loss of compartmentalisation characterises ARDS pathophysiology as compared to bacterial pneumonia. Indeed, the spreading of inflammatory response in all lung compartments has been clearly shown by numerous studies during ARDS [3, 25], by contrast, a similar but localised response is evidenced during bacterial pneumonia without ARDS [24]. Interestingly, the extension of inflammatory response to the whole lung during ARDS is not due to the spreading of infectious organisms. Similarly, the occurrence of septic shock during bacterial pneumonia is also due to the spillover of inflammatory mediators from lung as elegantly demonstrated by KURAHASHI et al. [26].

\section{Nosocomial pneumonia: a specific inflammatory response?}

For lower respiratory tract infection to occur, at least one of the following conditions must be present: 1) inoculation of organisms in sufficient number into the lower respiratory tract to overwhelm the host's defences; 2) alteration of the host defence mechanisms. Both factors probably contribute to the dramatically high incidence of pneumonia, particularly in patients under mechanical ventilation $(\sim 20 \%$ of patients who are ventilated for $>48 \mathrm{~h}$ ). Concerning the inflammatory response, the initial injury leading to intensive care unit admission will modify host's defences and their ability to respond to a subsequent insult as nosocomial pneumonia. For instance, functions of both monocyte/macrophage and neutrophil are modified in critically ill patients. Expression of human leukocyte antigen (HLA)-DR molecules on monocytes and bactericidal activity of blood neutrophils are impaired in critically ill patients $[27,28]$. Other modifications of innate immunity have been characterised in the murine peritonitis model: decreased expression of major histocompatibility complex molecules, decreased synthesis of nitric oxide, and resulting impaired bactericidal activity by alveolar macrophages. Similarly, in the same model, an impairment of the capability of blood neutrophils to migrate into alveolar spaces has been shown [29].

\section{Conclusion}

Inflammatory response to infectious pneumonia is one of the components of the innate immune response. This local inflammatory response can be easily monitored using bronchoalveolar lavage studies: soluble mediators of innate response, as cytokines, are retrieved, and inflammatory cells can be isolated and cultured.

\section{References}

1. Delves PJ, Roitt IM. The immune system. First of two parts. $N$ Engl J Med 2000; 343: 37-49.

2. Delves PJ, Roitt IM. The immune system. Second of two parts. $N$ Engl J Med 2000; 343: 108-117.

3. Monton C, Torres A. Lung inflammatory response in pneumonia. Monaldi Arch Chest Dis 1998; 53: 56-63.

4. Zhang P, Summer WR, Bagby GJ, Nelson S. Innate immunity and pulmonary host defense. Immunol Rev 2000; 173: 39-51.

5. Sibille Y, Reynolds HY. Macrophages and polymorphonuclear neutrophils in lung defense and injury. Am Rev Respir Dis 1990; 141: 471-501.

6. Crouch E, Wright JR. Surfactant proteins a and d and pulmonary host defense. Annu Rev Physiol 2001; 63: 521554.

7. Holter JF, Weiland JE, Pacht ER, Gadek JE, Davis WB. Protein permeability in the adult respiratory distress syndrome. Loss of size selectivity of the alveolar epithelium. $J$ Clin Invest 1986; 78: 1513-1522.

8. Haslett C. Granulocyte apoptosis and its role in the resolution and control of lung inflammation. Am $J$ Respir Crit Care Med 1999; 160: S5-S11.

9. Ware LB, Matthay MA. The acute respiratory distress syndrome. N Engl J Med 2000; 342: 1334-1349.

10. Kelley J. Cytokines of the lung. Am Rev Respir Dis 1990; 141: 765-788.

11. Nelson S. Novel nonantibiotic therapies for pneumonia: cytokines and host defense. Chest 2001; 119: 419S-425S.

12. Horvath CJ, Ferro TJ, Jesmok G, Malik AB. Recombinant tumor necrosis factor increases pulmonary vascular 
permeability independent of neutrophils. Proc Natl Acad Sci USA 1988; 85: 9219-9223.

13. Rezaiguia S, Garat C, Delclaux C, et al. Acute bacterial pneumonia in rats increases alveolar epithelial fluid clearance by a tumor necrosis factor-alpha-dependent mechanism. J Clin Invest 1997; 99: 325-335.

14. van der Poll T, Marchant A, Keogh CV, Goldman M, Lowry SF. Interleukin-10 impairs host defense in murine pneumococcal pneumonia. J Infect Dis 1996; 174: 994-1000.

15. Wagner JG, Roth RA. Neutrophil migration mechanisms, with an emphasis on the pulmonary vasculature. Pharmacol Rev 2000; 52: 349-374.

16. Cassatella MA, Gasperini S, Russo MP. Cytokine expression and release by neutrophils. Ann N Y Acad Sci 1997; 832: 233242.

17. Medzhitov R, Janeway C Jr. Innate immunity. $N$ Engl J Med 2000; 343: 338-344.

18. Hashimoto S, Kobayashi A, Kooguchi K, Kitamura Y, Onodera $H$, Nakajima $H$. Upregulation of two death pathways of perforin/granzyme and FasL/Fas in septic acute respiratory distress syndrome. Am J Respir Crit Care Med 2000; 161: 237-243.

19. Crestani B, Aubier M. Inflammatory role of alveolar epithelial cells. Kidney Int Suppl 1998; 65: S88-S93.

20. Greenberger MJ, Kunkel SL, Strieter RM, et al. IL-12 gene therapy protects mice in lethal Klebsiella pneumonia. J Immunol 1996; 157: 3006-3012.

21. Tazi A, Nioche S, Chastre J, Smiejan JM, Hance AJ. Spontaneous release of granulocyte colony-stimulating factor (G-CSF) by alveolar macrophages in the course of bacterial pneumonia and sarcoidosis: endotoxin-dependent and endotoxin-independent G-CSF release by cells recovered by bronchoalveolar lavage. Am J Respir Cell Mol Biol 1991; 4: $140-147$.

22. Bogdan C. Nitric oxide and the immune response. Nat Immunol 2001; 2: 907-916.

23. Kharitonov SA, Barnes PJ. Exhaled markers of pulmonary disease. Am J Respir Crit Care Med 2001; 163: 1693-1722.

24. Dehoux MS, Boutten A, Ostinelli J, et al. Compartmentalized cytokine production within the human lung in unilateral pneumonia. Am J Respir Crit Care Med 1994; 150: 710-716.

25. Bauer TT, Monton C, Torres A, et al. Comparison of systemic cytokine levels in patients with acute respiratory distress syndrome, severe pneumonia, and controls. Thorax 2000; 55: 46-52.

26. Kurahashi K, Kajikawa O, Sawa T, et al. Pathogenesis of septic shock in Pseudomonas aeruginosa pneumonia. J Clin Invest 1999; 104: 743-750.

27. Döcke WD, Randow F, Syrbe U, et al. Monocyte deactivation in septic patients: Restauration by INF- $\gamma$ treatment. Nature Med 1997; 3: 678-681.

28. Stephan F, Yang K, Tankovic J, et al. Impairment of polymorphonuclear neutrophil functions precedes nosocomial infections in critically ill patients. Crit Care Med 2002; 30: $315-322$.

29. Attalah HL, Azoulay E, Yang K, et al. Granulocyte colonystimulating factor enhances host defenses against bacterial pneumonia following peritonitis in nonneutropenic rats. Crit Care Med 2002; 30: 2107-2114. 